Reprod. Nutr. Dévelop., 1988, 28 Suppl. n 1, 135-136

\title{
Estimation par le CR-EDTA des variations intra-journalières du volume de contenu digestif dans le réticulo-rumen du mouton
}

R. BAUMONT, C. PONCET (*)

Unité de l'Ingestion

(*) Unité de la dynamique de la Digestion

Station de Recherches sur la Nutrition des Herbivores

I.N.R.A., Theix, 63122 Ceyrat, France.

Summary. The use of $\mathrm{Cr}$-EDTA for estimation of intradiurnal variations of the reticulo-ruminal fill was studied using six sheep fed ad lib. grass hay twice a day. The results are compared with those obtained by manual emptying of the reticulo-rumen.

La quantité de contenu digestif présente à un instant donné dans le reticulo-rumen ( $R R$ ) peut être mesurée après abattage ou par vidage complet sur des animaux munis de canules. Nous avons étudié l'intérêt d'un marqueur de la phase liquide pour estimer les variations intrajournalières du volume de contenu digestif dans le RR et l'avons comparé au vidage manuel.

Matériel et méthodes. Six moutons $(58 \pm 2 \mathrm{~kg})$ ont reçu une infusion continue de Cr-EDTA (Binnerts et al., 1968). A partir du $4^{\mathrm{e}}$ jour d'infusion, un prélèvement de contenu de rumen a été effectué toutes les $6 \mathrm{~h}$ pendant $72 \mathrm{~h}$ afin d'obtenir 12 prélèvements couvrant l'ensemble du nycthémère. Après arrêt de l'infusion 15 prélèvements répartis sur $36 \mathrm{~h}$ ont été effectués afin de mesurer le taux de renouvellement de la phase liquide. Durant les deux semaines suivantes quatre vidages manuels du RR ont été réalisés sur chaque animal (avant et après les deux repas principaux). A un instant $T$ le volume $V(T)$ de la phase liquide présente dans le RR est estimé ainsi : $V(T)=Q /(k \times C(T))$ avec $k=$ taux de renouvellement horaire du $\mathrm{Cr}$-EDTA (supposé constant); $\mathrm{Q}=$ quantité de marqueur infusée par heure ; $C(T)=$ concentration en $\mathrm{Cr}$-EDTA à l'instant $\mathrm{T}$. Les valeurs ainsi calculées sont comparées aux valeurs de la partie liquide du contenu (contenu total-matière sèche) mesurées lors des vidages (48 comparaisons). Les mesures ont été réalisées au cours de deux périodes expérimentales, les animaux étant alimentés à volonté en accès continu avec un foin de prairie tardif (digestibilité de la MS de 50\%) : P1 : deux distributions de foin à $9 \mathrm{~h}$ et à $17 \mathrm{~h}$ et éclairage $12 \mathrm{~h}$ sur $24 ; \mathrm{P} 2$ : deux distributions à $9 \mathrm{~h}$ et à $21 \mathrm{~h}$ et éclairage continu. Les quantités ingérées et les activités alimentaires ont été enregistrées durant ces deux périodes (Baumont et al., 1988).

Résultats et discussion. La distribution du foin entraîne toujours une forte augmentation (fig. 1) du volume de la phase liquide du RR dans les deux heures suivantes. Ces deux repas représentent respectivement 82,87 et $83 \%$ du total des durées d'ingestion ( $309 \pm 51 \mathrm{~min}$ ), des quantités ingérées ( $1094 \pm 140 \mathrm{~g}$ de $\mathrm{MS})$ et de l'eau consommée $(2,8 \pm 0,51)$. La teneur en MS du contenu du RR, minimale avant la distribution du matin et maximale après le repas du soir varie entre $10,6 \pm 1,3$ et $13,8 \pm 0,7 \%$ 

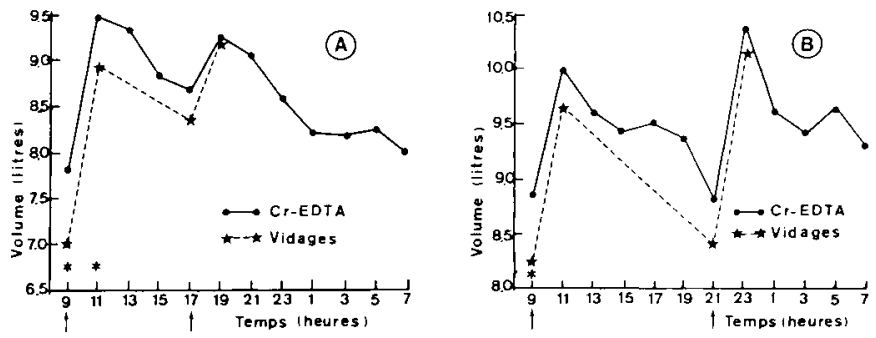

FIG. 1. - Evolution au cours du nycthémère du volume de la phase liquide du RR sur 6 moutons recevant un foin de prairie en deux distributions à $9 h$ et $17 h$ (A) ou à $9 h$ et $21 h$ (B). Les ( $\uparrow$ ) indiquent les distributions et les (*), une différence significative $(P<0,05)$ entre les deux méthodes.

Les volumes de contenu estimés par le Cr-EDTA ont tendance à être plus élevés que ceux mesurés lors des vidages, en moyenne de 0,41 I soit $5,1 \%$ $(P>0,05)$, cet écart étant plus important avant la distribution du foin (fig. 1). Sur l'ensemble des 48 comparaisons, les écarts entre les mesures présentent une forte variabilité (écart-type de 0,62I) qui peut s'expliquer par le fait qu'elles n'ont pas été réalisées simultanément, l'écart-type journalier des quantités ingérées étant de $72 \mathrm{~g}$ de MS. Toutefois, si on considère les 8 valeurs correspondant à une série de comparaisons ( 6 moutons) la variabilité des écarts entre les deux méthodes est plus faible (écart-type de 0,29 I). La répartition du marqueur dans le RR a été homogène, le coefficient de variation entre quatre lieux de prélèvements (zone antérieure du RR, postérieure, sac ventral et dorsal au niveau de la canule) n'excédant pas 4,9\%. En revanche, il est possible que lors des vidages manuels, surtout avant les distributions de foin, la teneur en MS étant plus faible, il soit difficile d'extraire tout le liquide. A l'inverse, Egan et al. (1983) observent une sous-estimation de 7,8 à $15,9 \%$ avec le $\mathrm{Cr}$-EDTA par rapport aux valeurs mesurées après abattage.

L'estimation des variations de volume du RR par le Cr-EDTA a été meilleure en P2 (les grands repas étant espacés de $12 \mathrm{~h}$ ) (fig. 1). Celà peut être dû au fait que en P1 (les grands repas débutant à $9 \mathrm{~h}$ et à $17 \mathrm{~h}$ ), le taux de renouvellement de la phase liquide n'est pas constant au cours du nycthémère $(8,15 \pm 1,15$ le jour vs. $6,17 \pm 1,02 \%$ la nuit, $P<0,05$ ) et les animaux ruminent plus la nuit $(386 \pm 24$ vs $258 \pm 45 \mathrm{mn}$ le jour, $\mathrm{P}<0,05)$. De telles différences ne sont pas observées en P2.

En conc/usion, l'utilisation du Cr-EDTA pour étudier les variations intrajournalières du volume de la phase liquide du RR est satisfaisante, et d'autant meilleure que le mode d'alimentation permet une répartition plus régulière des activités alimentaires au cours du nycthémère.

Remerciements. - à J. M. Luginbuhl (North Carolina State University, Department of Animal Science) pour la discussion du protocole.

Baumont R., Dulphy J. P., Andrieu J. P., 1988. Reprod. Nutr. Dévelop., 28, 573-588.

Binnerts W. T., van't Klooster A. T., Frens A. M., 1968. Vet. Rec., 82, 470.

Egan J. K., Pearce G. R., Doyle P. T., Thomas R., 1983. Aust. J. agric. Res., 34, 307-315. 